\title{
The effect of food deprivation on human resolving power
}

\author{
Noa Zitron-Emanuel ${ }^{1} \cdot$ Tzvi Ganel $^{1}$
}

Published online: 3 May 2017

(C) Psychonomic Society, Inc. 2017

\begin{abstract}
The feeling of hunger is an inseparable part of people's daily lives. It has been established that hunger, caused by food deprivation, influences people's physiological and emotional state and their everyday behavior. Yet, it remains unclear whether and in which manner food deprivation affects the way people perceive their environment. In two experiments, we examined the effects of food deprivation on the perceptual resolution of food portion size. We calculated Just Noticeable Differences (JNDs) to measure sensitivity to detect the smallest difference between two stimuli of different sizes. Participants' resolution in both experiments was higher to detect changes in food size compared with baseline when they were hungry due to a short period of food deprivation. Food deprivation did not lead to any biases in the average perception of food size. The results show that food deprivation changes the way people perceive their environment. We discuss the possible role of attention in mediating the effect of food deprivation on the visual resolution of food size.
\end{abstract}

Keywords Hunger · Food deprivation · Psychophysics · Visual perception $\cdot$ Motivational effects $\cdot$ Spatial resolution

For several decades, psychologists have looked into possible effects of motivational factors on human perception and cognition. Findings were largely inconsistent; several studies yielded results suggesting that motivational factors influence perception, as demonstrated by Bruner and Goodman (1947),

Tzvi Ganel

tganel@bgu.ac.il

1 Department of Psychology, Ben-Gurion University of the Negev, Beer-Sheva 84105, Israel who found that poor children perceive the size of a coin as bigger compared with rich children. However, those studies were heavily criticized due to methodological issues as well as for the theoretical aspects of the drawn conclusions (Tajfel, 1957; Veltkamp, Aarts, \& Custers, 2008).

Previous related literature has mainly focused on the effects of different descriptive variables, such as the participant's gender, age, or body mass index (BMI), on average perceived size, whereas fewer studies have looked into the effects of motivational states, such as hunger and food-deprivation (Beasley, Hackett, Maxwell, \& Stevenson, 2004). We focus on the effect of hunger, caused by mild food deprivation, on human perceptual resolution.

The feeling of hunger is an inseparable part of our daily lives. It has been widely established that hunger can have a strong influence on people's physiological and emotional state and on their everyday behavior (Berridge, 2004; Loewenstein, 1996). Despite its major impact on our daily lives, it remains unclear whether and in which manner hunger affects the way people perceive their external environment. Lazarus, Yousem, and Arenberg (1953) reviewed several studies that looked at the relationship between hunger and perception and pointed out that some of these studies did not actually measure perception. Virtually, all previous studies on motivational effects (including hunger) on size perception have focused on the effect of motivational variables on biases in average perceived size (Witt \& Dorsch, 2009). A recent study asked participants to estimate the size of a water glass, under fluid deprivation, and showed that deprived participants perceived a glass of water as bigger compared with nondeprived participants (Veltkamp et al., 2008). Yet, the literature in the field is inconclusive, probably due to the large variability across experimental methods and stimuli used. For example, Kral, Roe, and Rolls (2004) served participants with different meals (breakfast, lunch, and dinner) of varying portion sizes 
$(100 \%, 150 \%, 200 \%)$ across 3 weeks. Participants were asked to rate the perceived size of the first and last dish of each meal - representing conditions of hunger and satiety. No significant differences were found between the conditions.

In a seminal study that looked at the effects of food deprivation on perception, Lazarus and his colleagues (1953) provided initial evidence that mild food deprivation may lead to a decreased threshold for shape identification of food-related stimulus. More recently, Radel and Clement-Guillotin (2012) showed that food-deprived participants were more likely to identify briefly presented food-related words compared with nondeprived participants. Note, however, that the last study focused on the effect of food deprivation on semantic word identification rather than on visual resolution of foodrelated stimuli.

The present study examined the effects of hunger on the perceptual resolution of physical food size. To this purpose, we used the classic method of constant stimuli tailored to the domain of food size perception. We calculated JNDs to measure the sensitivity to detect the smallest difference between two stimuli of differing sizes and calculated possible biases in food portion size perception.

\section{Experiment 1}

We examined the effects of mild food deprivation on participants' sensitivity to detect changes in the size of a food-related stimulus compared with neutral, control stimuli. We hypothesized that food-deprived participants would be more sensitive to differences in the size of a food stimulus compared with simple-shape stimulus and compared with nondeprived participants. In other words, it was predicted that JNDs for food related-stimuli would be smaller compared with baseline in the food-deprived group.

\section{Methods}

\section{Participants}

Seventy-two undergraduate female students (age range: 18-29 years, mean $=22.98, \mathrm{SD}=1.53$ ) from Ben-Gurion University of the Negev, participated in the study for credit in a psychology course or received the equivalent of $\$ 6$ for their participation. All participants had normal or corrected-to-normal vision; 39 participants were randomly allocated to the fooddeprivation condition; the other 33 participants were allocated to the nondeprivation condition. Data from five participants ( 3 food-deprived, 2 nondeprived participants) were excluded from the analyses due to low (smaller than 70\%) values of Goodness-of-Fit. Data from four additional participants (1 food-deprived, 3 nondeprived) self-reported as being clinically diagnosed with an eating disorder also were excluded.
Participants gave an informed, written consent before their participation in the experiment, which was approved by the Ethics Committee of the Psychology Department at BenGurion University of the Negev. The experiment lasted approximately 40 minutes.

\section{Stimuli}

Stimuli consisted of high-resolution color images of food and neutral stimuli or round contours of those stimuli as control (Fig. 1). We used images of a chocolate chip cookie (food stimulus), a tennis ball (neutral stimulus), and a circle contour (simple-shaped stimulus). Images were edited using Photoshop CS by cutting the images into a nearly perfect circle. The resulting stimulus set included a base, standard stimulus, and 12 additional reference stimuli, smaller or bigger than the 95-mm standard stimulus in constant $1 \mathrm{~mm}$ intervals. All stimuli were presented on a white background and were viewed from $60-\mathrm{cm}$ distance on a 19 " computer screen.

\section{Design and procedure}

Participants who were assigned to the food-deprivation condition were asked not to eat at least 3 hours before the experiment. Those assigned to the nondeprived condition were asked to eat during the hour before the experiment.

Participants were asked to choose the smaller image of the two images presented on the screen by pressing the appropriate keyboard key. The experiment began with two practice trials that were discarded from further analyses and continued with four experimental blocks: block A consisted of a pair of food stimuli-a chocolate chip cookie standard image and a chocolate chip cookie reference image; block B consisted of a pair of simple-shaped stimuli-a circle-shaped standard image and a circle-shaped reference image. These two blocks were designed to measure JNDs or perceptual resolution (Fig. 1). Block $\mathrm{C}$ consisted of a food stimulus and a simpleshaped stimulus - a chocolate chip cookie standard image and a circle-shaped reference image; and block D consisted of a neutral stimulus and a simple-shaped stimulus - a tennis ball standard image and a circle-shaped reference image. These two blocks were designed to measure PSEs to look for potential effects of perceptual bias. All blocks were randomly ordered across participants.

Participants were presented with a pair of images in each trial - the standard image and 1 of the 12 reference images. The images were presented at opposite heights and sides to avoid participants of using height cues. Relative locations were counterbalanced across trials (left-up, left-down, rightup, right-down). A 500-msec fixation dot appeared between trials. Within each block, each of the 12 standard-reference combinations was randomly repeated 16 times, resulting in 192 trials per block, and 768 trials overall. 
(a)

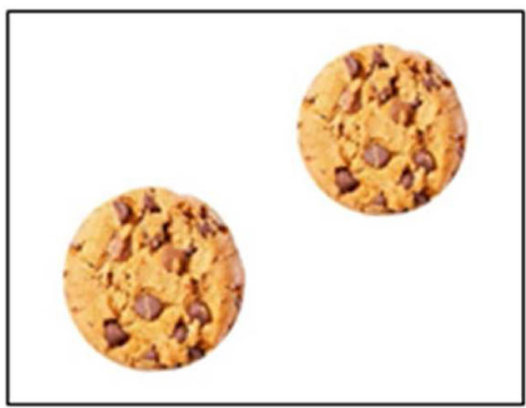

(c)

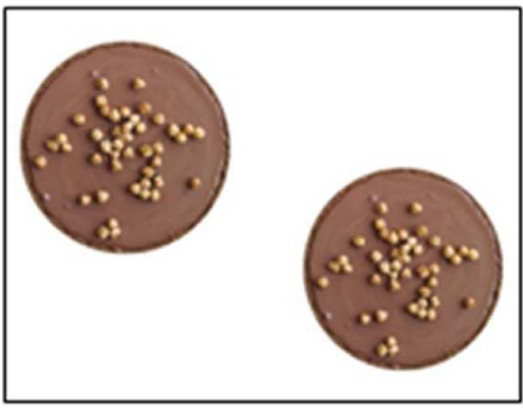

Fig. 1 Stimuli used in Experiments 1 and 2. Depicted are the standard (baseline) stimulus and one of the reference stimuli: (a) chocolate chip cookie standard image and chocolate chip cookie reference-image used in Experiment 1; (b) simple circle shape standard image and reference

Following the experimental session, participants were asked to complete the EAT-26 (Eating Attitudes Test) questionnaire as a screening tool for eating disorders symptoms. Higher scores indicate greater pathology, and a score greater than 20 is recommended as the clinical cutoff (Garner, 2004).

\section{Data analysis}

Blocks A and B were used to calculate JNDs for the food and control stimuli. We first calculated for each of the reference stimuli the proportion of the number of trials in which it was perceived as smaller than the standard stimulus compared with the total number of trials. We then fitted the data on a sigmoid function (perceptual function), which plotted those proportions against the values of the stimuli, ranging from $8.9 \mathrm{~cm}$ to $10.1 \mathrm{~cm}$. Finally, the JND was calculated by dividing the stimulus range between $25 \%$ and $75 \%$ correct discrimination (this range is termed - "the area of uncertainty," an area in which the stimulus size is not perceived as distinctively different from the standard stimulus) by two (Namdar, Avidan, \& Ganel, 2015).

Blocks C and D were used to calculate PSEs for the chocolate chip cookie and the tennis ball. The same calculation as described above was performed for each participant, only by identifying the stimulus value that corresponds to $50 \%$ correct discrimination (at this stimulus value the participant perceived (b)

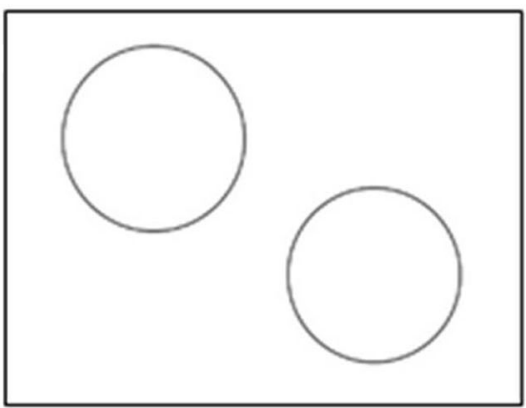

(d)

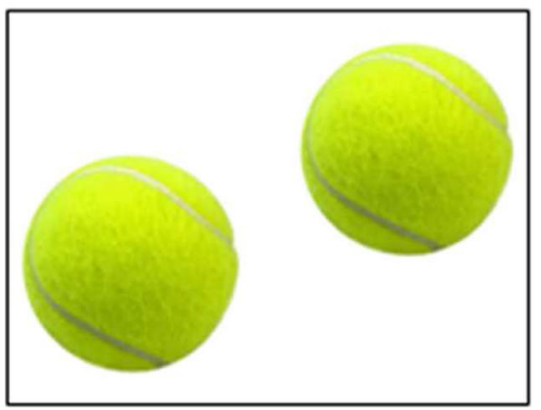

image; (c) chocolate pie standard image and chocolate pie reference image used in Experiment 2; (d) tennis ball standard image and tennis ball reference image used in Experiment 2

the reference stimulus as equal to the standard, which represents its perceived size).

Note that the data from blocks $\mathrm{C}$ and $\mathrm{D}$ can theoretically be used to calculate JNDs, but the interpretation and the meaning of these measures is quite problematic and not effective. The design in blocks $\mathrm{C}$ and $\mathrm{D}$ does not measure subjects' resolution in detecting the slightest difference between two almostidentical food stimuli (a within-category comparison), but rather subjects' ability to tell the difference between the diameter of a food stimulus and the diameter of a different object (a between-category comparison).

To validate the fit of the participant's pattern to the sigmoid function, we computed Goodness of Fit (GOF) scores for each block. Participants, who showed GOF $<0.7$ in one of the blocks, were excluded from further analysis. The total averages (SD) of GOF scores were 0.95 (0.05), 0.94 (0.05), 0.93 (0.05), and $0.94(0.04)$ in blocks $\mathrm{A}, \mathrm{B}, \mathrm{C}$, and $\mathrm{D}$, respectively.

\section{Results and discussion}

JND scores were submitted to a 2-factor mixed effects analysis of variance (ANOVA). It consisted of a within-subject factor of stimulus type (food stimulus vs. baseline) and one between-subjects factor of group (food deprived vs. nondeprived). The results are presented in Fig. 2. JNDs for the food-deprived group were smaller for the food stimulus compared with the control stimulus. The nondeprived group 


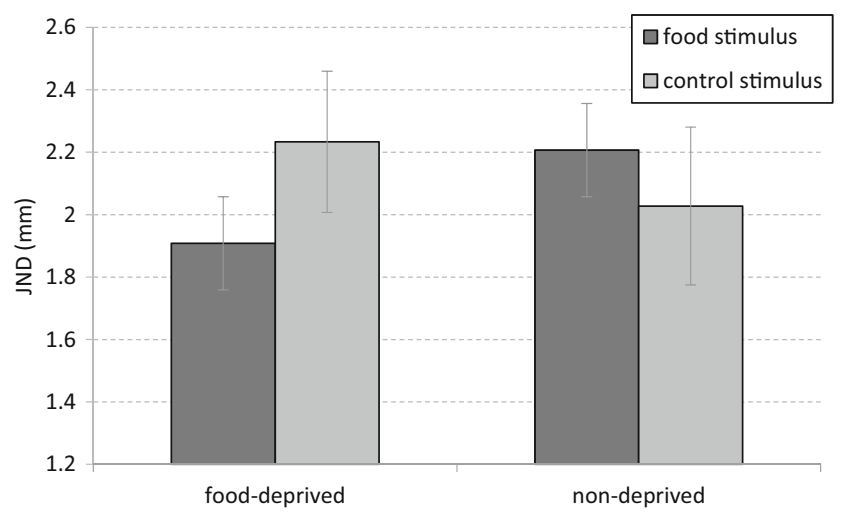

Fig. 2 JND scores for the food stimulus and the baseline stimulus, for food-deprived, and for nondeprived participants in Experiment 1. Perceptual sensitivity for food stimuli in the food deprived group was significantly higher than baseline. No statistical difference was found for the nondeprived group. Error bars represent $95 \%$ confidence intervals for mixed-design ANOVAs

did not show such an increase in performance for the foodrelated stimulus.

The EAT-26 questionnaire consisted of 26 items; Cronbach's alpha is $\mathbf{0 . 8 3 3}$, which indicates a high level of reliability and internal consistency. The average measure ICC for PSE was 0.749 with a $95 \%$ confidence interval from 0.585 to $0.848(\mathrm{~F}(62,62)=3.983, p<0.0001)$ and the average measure ICC for JND was 0.699 with a $95 \%$ confidence interval from 0.503 to $0.818(\mathrm{~F}(62,62)=3.324, p<0.0001)$. This states that $74.9 \%$ of the variance in the PSE scores and $69.9 \%$ of the variance in the JND scores could be accounted for, indicating an acceptable level of intra-subject reliability. The main effects of stimulus type $[\mathrm{F}(1,61)=0.369, p>0.05$, $\left.\eta_{\mathrm{p}}^{2}=0.006\right]$ and group $\left[\mathrm{F}(1,61)=0.042, p>0.05, \eta_{\mathrm{p}}^{2}=\right.$ 0.001 ] were not significant, suggesting that there were no statistical differences between JNDs for food and for baseline stimuli, nor between the deprived and nondeprived groups.

More importantly, the interaction between stimulus type and group was significant $\left[\mathrm{F}(1,61)=4.416, \mathrm{p}<0.05, \eta_{\mathrm{p}}{ }^{2}=\right.$ 0.068 ], indicating that food-deprived, compared with nondeprived participants, showed a different pattern of JND scores to food compared with baseline, control-stimuli. Specific comparisons revealed that JNDs for food stimuli were significantly lower compared with baseline in the fooddeprived group $[\mathrm{t}(34)=2.95, p<0.01]$ but not in the nondeprived group [ $\mathrm{t}(27)=0.777, p>0.05]$. Although there was a trend indicating smaller JNDs for food stimuli and larger JNDs for baseline stimuli, specific comparison did not show significant effects either for the baseline $[\mathrm{t}(61)=$ $0.874, p>0.05]$ or for the food stimuli $[\mathrm{t}(61)=1.083, p>$ $0.05]$ when the food-deprived group was compared with the nondeprived group (Fig. 2).

We also performed a Bayesian repeated measures ANOVA, aimed to select the best-fitting ANOVA model to the data, based on the Bayes Factor (BF) statistic. BFs were calculated using the JASP software package (JASP Team, 2016). In contrast to the classical ANOVA reported above, the Bayesian analysis failed to support any of the substantial models. Specifically, the null model performed better than any other model. Specifically, the best-fitting model included a main effect of stimulus type; however, even this model did not provide a better fit than the null model $\left[\mathrm{BF}_{10}=0.318\right]$. We hypothesize that these nonsignificant effects in the specific comparisons and in the Bayesian analysis are due to the relatively low statistical power inherent to the between-subject design used in Experiment 1. Experiment 2 was designed accordingly to extend and replicate the results of the current experiment using a more powerful experimental design.

As for the JND scores, we also calculated PSEs for food and neutral stimuli for the two groups. The main effects of stimulus type $\left[\mathrm{F}(1,61)=0.182, p>0.05, \eta_{\mathrm{p}}{ }^{2}=0.003\right]$ and group $\left[\mathrm{F}(1,61)=0.033, p>0.05, \eta_{\mathrm{p}}{ }^{2}=0.011\right]$ were not significant. The interaction between stimulus type and group also was not significant $\left[\mathrm{F}(1,61)=0.673, p>0.05, \eta_{\mathrm{p}}{ }^{2}=0.001\right]$, indicating that food-deprived participants in general did not differ from nondeprived participants in terms of their average perceptual biases for food portion size. The Pearson correlations between JND scores for food stimuli and the EAT-26 scores $[\mathrm{r}=0.004, p>0.05]$ and between PSE scores for food-stimuli and the EAT-26 scores $[\mathrm{r}=0.012, p>0.05]$ were not statistically significant.

These results show that mild amount of food depravation leads to an increased spatial resolution for food-related stimuli. Results show that JNDs were smaller for food-related stimuli compared with baseline in the food-deprived group but not in the nondeprived group. This evidence supports the hypothesis that JNDs for the size of food stimuli are affected by objective physiological factors.

We propose that the results of Experiment 1 suggest that hunger, triggered by mild food depravation, has a significant effect on human resolving power for food-related stimuli. However, although the relationship between food deprivation and hunger has been well established (Benau, Orloff, Janke, Serpell, \& Timko, 2014; Loewenstein, 1996), the design of Experiment 1 did not support a direct link between food deprivation and hunger, because the subjective feeling of hunger was not directly assessed.

Before embracing the idea that food deprivation led to increased sensitivity for food-related stimuli, a different interpretation also should be considered. In particular, the food stimuli in Experiment 1 were richer in texture and in fine visual details and complexity than the simple-shape stimulus used as baseline. Therefore, it could be argued that food deprivation led to increased sensitivity in attending to the fine details of a stimulus, rather than to specific increased sensitivity to foodrelated stimuli. To answer this potential concern, a control stimulus with rich textures and high resolution was 
used in Experiment 2 instead of the simple-shaped circle used in Experiment 1.

Finally, we note that the between-group experimental design used in Experiment 1 could have led to incidental between-subjects differences, which could decrease the statistical power of the experiment. Such statistical noise could have accounted for the nonsignificant pattern of results revealed in the specific comparisons analysis across the two experimental groups as well as the results of the Bayesian analysis. Experiment 2 therefore was designed as a withinsubject experiment to increase statistical power. In addition, as in most previous studies in the field of food perception, only female subjects were included in Experiment 1. Research in the field typically focused on females probably due to their elevated vulnerability to food-related disorders, such as Bulimia Nervosa (Ptacek et al., 2016). In Experiment 2 , we included male and female participants to generalize the results of Experiment 1 across gender. This also allowed us to compare females and males in respect to their sensitivity to food stimuli.

Experiment 2 was designed to extend and generalize the results of Experiment 1 along several aspects. First, we established the role of hunger in mediating resolution for food size by including a direct measure of perceived hunger. Second, to address the issue of incidental between-group differences and to extend the generality of the results across gender, Experiment 2 was designed as a within-subject experiment, and similar numbers of male and of female subjects participated in the experiment. Finally, a different type of food and neutral stimuli were used in Experiment 2 to extend and to generalize the findings across different situations.

\section{Experiment 2}

Each of the participants was included in both the fooddeprived and nondeprived conditions, in a counterbalanced order and in separate days. Furthermore, Experiment 2 was designed to address the issue of potential differences in visual details between the food-related stimulus and the control stimulus and to generalize the results of Experiment 1 across male and female subjects.

\section{Methods}

The design of Experiment 2 was similar to that used in Experiment 1, with several changes described hereinafter.

\section{Participants}

Eighty-eight undergraduate students (age range: 18-31 years, mean $=23.36, \mathrm{SD}=2.35 ; 45$ females and 43 males , who did not participate in Experiment 1, took part in the experiment.
All participants underwent the two experimental conditions: food-deprivation and nondeprivation. Condition order was counterbalanced between subjects. Data from eight participants were excluded from the analyses due to low $(<70 \%)$ values of Goodness-of-Fit. Two participants had very noisy data, with JNDs higher than 3 SD than average, and their data were excluded. Data from one participant who self-reported to be clinically diagnosed with an eating disorder also were excluded.

\section{Stimuli}

The experiment consisted of an 11-cm diameter standard stimulus and 12 references stimuli, varying between $10.4 \mathrm{~cm}$ and $11.6 \mathrm{~cm}$ diameters. We used images of a chocolate pie and a tennis ball (Fig. 1).

\section{Design and procedure}

This experiment included two sessions, separated by 1-6 days, one for each experimental condition described in Experiment 1. Prior to the experimental session, participants were asked to rate how hungry they feel, on a subjective "hunger-scale" (rated on a 7 points scale: 1 = "replete," not hungry at all, 7 = "starving," extremely hungry).

\section{Data analysis}

Data were fitted on a sigmoid function (perceptual function), which plotted those proportions against the values of the stimuli, ranging from $10.4 \mathrm{~cm}$ to $11.6 \mathrm{~cm}$. The total average (SD) of GOF scores were 0.94 (0.06) and 0.93 (0.06), for the fooddeprived and nondeprived conditions, respectively.

\section{Results and discussion}

An initial analysis that included gender as a between-subject factor did not reveal a main effect of gender or any interactions related to gender. We therefore collapsed the results across females and males in subsequent analyses. JND scores were submitted to a two-way repeated measures analysis of variance (ANOVA) with the within-subject factors of stimulus type (food vs. baseline) and condition (food deprived vs. nondeprived). As shown in Fig. 3, the results provide a close replication of the results of Experiment 1; higher spatial sensitivity was found for the food related stimulus compared with baseline in the food-deprived condition. Such differences were not observed in the nondeprived condition.

A main effect of stimulus type $[\mathrm{F}(1,76)=7.225, p<0.01$, $\left.\eta_{\mathrm{p}}{ }^{2}=0.087\right]$ was found, with overall larger JNDs for the baseline compared with the food stimulus. The main effect of condition $\left[\mathrm{F}(1,76)=0.131, p>0.05, \eta_{\mathrm{p}}{ }^{2}=0.002\right]$ was not significant. More importantly, a significant interaction 


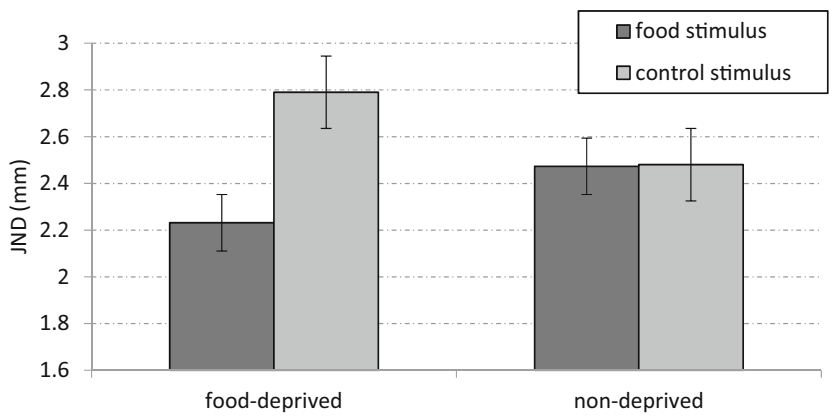

Fig. 3 JND scores for food-related and control stimuli, in the fooddeprived and the nondeprived conditions in Experiment 2. As in Experiment 1, under food deprivation, perceptual resolution was higher for the food-related compared with the control stimulus. Resolution did not statistically differ for the two stimulus types in the nondeprived condition. Error bars represent $95 \%$ confidence intervals for repeated measures ANOVAs

was found between stimulus type and condition $[\mathrm{F}(1,76)=$ 15.062, $\left.p<0.01, \eta_{\mathrm{p}}{ }^{2} \eta^{2} \mathrm{p}=0.165\right]$, indicating that food deprivation led to a different pattern of sensitivity for the foodrelated compared with the control stimuli. Specific comparisons showed that JNDs were significantly lower for the foodrelated stimulus compared with the baseline stimulus in the food-deprived condition [ $\mathrm{t}(76)=4.099, p<0.0001, d=0.52]$. JNDs for the food-related stimulus were not statistically different than baseline in the nondeprived condition $[\mathrm{t}(76)=$ $0.061, p>0.05, d=0.24]$. Interestingly, JNDs were significantly lower for the food stimulus in the food-deprived compared to the nondeprived condition [ $\mathrm{t}(76)=2.067, p<0.05, d$ $=0.25]$. Conversely, JNDs were significantly higher for the baseline stimulus in the food-deprived compared with the nondeprived condition [ $\mathrm{t}(76)=2.593, p<0.05, d=0.25]$. In the Bayesian ANOVA, the best-fitting model included condition, stimulus type, and their interaction as predictors $\left[\mathrm{BF}_{10}=\right.$ 16.13]. Moreover, $\mathrm{BF}_{10}$ was 1.28 for a model with only two main effects. Accordingly, the model that included the interaction term was 12.6 times more likely compared with the model that included only the two main effects.

These findings extend and replicate the results found in Experiment 1 and show again that perceptual resolution for the size of food-related stimuli is modulated by food deprivation. The specific comparisons showed that while food deprivation led to a significant increase in perceptual resolution for food-related stimuli, it also led to a significant decrease in perceptual resolution for the neutral, food-unrelated stimulus. These findings suggest that attentional processes may have modulated the effect of food deprivation on perceptual resolution in a similar manner to the way attention has been shown to affect performance under spatial attention tasks (Montagna, Pestilli, \& Carrasco, 2009). In particular, it can be argued that under food deprivation, participants allocate increased attentional resources to relevant, food related stimuli but also allocate less attentional resources (compared with the nondeprived condition) to food-unrelated, less attended neutral stimuli. We further elaborate about the possible role of attention in the General discussion section.

As expected, subjective hunger ratings were significantly higher in the food-deprived condition compared with the nondeprived condition, (5.18 and 2.27 , respectively; $\mathrm{t}(76)=$ 26.161, $p<0.0001, d=3.71)$. These results establish the relationship between hunger and food deprivation and confirm the experimental manipulation. We note, however, that such a relationship cannot confirm causality.

\section{General discussion}

The purpose of the current study was to examine the effect of mild hunger caused by food-deprivation on spatial resolution. The results show that motivational states can lead to an increased spatial resolution for domain-relevant stimuli. More particularly, participants' perception was more sensitive to differences in food size, when they were hungry following a mild period of food deprivation. The fact that mild food deprivation increases human resolution power for images of food-related stimuli presented on a computer screen provides a strong indication for the generality of the effect.

\section{How food deprivation affects visual resolution: a possible role for attention}

The current findings extend previous research in the field of size perception, concerned mainly with perceptual biases (difference between perceived food size and real food size) and attentional biases (Veltkamp et al., 2008). Previous research has shown that visual attentional processes could be modulated by motivational states that dictate goals and incentives. For example, Mogg, Bradley, Hyare, and Lee (1998) used the dot probe task to investigate whether hunger is associated with attentional and preattentive biases for food stimuli. Results showed a significant increase in selective attention to foodrelated words for food-deprived compared with nondeprived participants. These findings indicate attentional vigilance for stimuli that are relevant to the subject's psychological state (Piech, Pastorino, \& Zald, 2010). Similar findings were found among restrained eaters (Hollitt, Kemps, Tiggemann, Smeets, \& Mills, 2010; Neimeijer, de Jong, \& Roefs, 2013). Therefore, stimuli of relevant significance to the motivational state can increase arousal and potentially attract more attention compared with neutral stimuli (Balcetis \& Dunning, 2006; Engelmann \& Pessoa, 2007; Gu, Liu, Kyritsis, \& Bondy, 2009; Piech et al., 2010).

We note, however, that unlike the vast majority of studies in the field of attention, that traditionally examined the allocation of spatial attention in situations in which attended and unattended stimuli are simultaneously 
presented within the same visual framework, the present study applied a different experimental design. More particularly, in the present set of experiments, the foodrelated and the food-unrelated stimuli were presented at different trials and at different time points. At a particular point in time, therefore, selective attention could always be fully allocated to the target stimulus. However, it is still possible that when participants were food deprived, they engaged larger attentional resources in trials in which food-related stimuli were presented compared with neutral trials. It therefore is intriguing to speculate that under the experimental settings of the present experiments, the greater amount of attention allocated to food stimuli led to an increase in spatial resolution. In a similar vein, studies of spatial selective attention showed that selective attention can lead to an increase in perceptual resolution to attended compared with unattended stimuli (Carrasco, Williams, \& Yeshurun, 2002; Piech et al., 2010). Such an increase in spatial resolution has been suggested to result from the modulation of spatial frequency tuning neural populations across the visual field (Carrasco et al., 2002). Although spatial selective attention was not directly examined in the current study, it is possible that attentional mechanisms mediated the effects of food deprivation on visual resolution. As in studies of spatial attention that showed that the processing of attended stimuli is enhanced compared with unattended stimuli (Montagna et al., 2009), the results of Experiment 2 showed that food deprivation led to an increase in resolution for food related stimuli but also to a decrease in resolution for foodunrelated stimuli, which could be therefore regarded as "less attended" compared with food-related stimuli. Therefore, it is possible that the effects of food deprivation on performance in the present study were mediated by the allocation of attention (for a discussion, see Firestone \& Scholl, 2015). However, due to the nature of the current experimental design, which did not directly manipulate attention, the possible relevance of selective attention to the present findings should be clarified in future research.

Unlike the effect food deprivation had on visual resolution in the present investigation, food deprivation did not lead to any distortions or biases in the average perception of food size. The scarce research in the field showed conflicting findings. This finding is congruent to several earlier studies; Carter and Schooler (1949) found no significant differences between rich and poor children's perception of coin size. Conversely, several studies have reported overestimation of stimuli size under certain motivational states and, more specifically, overestimation of food size following food-deprivation or under dietary states (Bruner \& Goodman, 1947; Neimeijer et al., 2013; Piech et al., 2010; Veltkamp et al., 2008). Accurate perception can be critical for one's survival. This may explain why motivational states or factors would not always lead to perceptual biases or distortions; rather they may more consistently sharpen people's perceptual sensitivity to increase processing efficiency (Tajfel, 1957).

\section{Conclusions}

The results of the present study show that food deprivation leads to an increased resolution power for food stimuli. In addition, the present study provides a basic psychophysical method to assess differences in size perception under different motivational states. By systematically investigating the effect of food deprivation and hunger on food size - perception in laboratory settings, it would be possible to gain further insights on how perception in normal subjects and in clinical populations suffering from eating disorders is modulated by motivational factors. Understanding such possible differences might help to shed a new light on the mechanisms that underlie normal perceptual processing of food-related stimuli.

Acknowledgments The authors thank Yoav Kessler and Daniel Algom for their help and advice.

\section{References}

Balcetis, E., \& Dunning, D. (2006). See what you want to see: Motivational influences on visual perception. Journal of Personality and Social Psychology, 91(4), 612-625. doi:10.1037/ 0022-3514.91.4.612

Beasley, L. J., Hackett, A. F., Maxwell, S. M., \& Stevenson, L. (2004). The effect of a dietary preload on estimation of usual food portion size by photograph in female volunteers. Journal of Human Nutrition and Dietetics, 17(3), 219-225. doi:10.1111/j.1365-277X. 2004.00515.x

Benau, E. M., Orloff, N. C., Janke, E. A., Serpell, L., \& Timko, C. A. (2014). A systematic review of the effects of experimental fasting on cognition. Appetite, 77, 52-61. doi:10.1016/j.appet.2014.02.014

Berridge, K. C. (2004). Motivation concepts in behavioral neuroscience. Physiology and Behavior, 81(2), 179-209. doi:10.1016/j.physbeh. 2004.02.004

Bruner, J. S., \& Goodman, C. C. (1947). Value and need as organizing factors in perception. Journal of Abnormal Psychology, 42(1), 3344. doi: $10.1037 / \mathrm{h} 0058484$

Carrasco, M., Williams, P. E., \& Yeshurun, Y. (2002). Covert attention increases spatial resolution with or without masks: Support for signal enhancement. Journal of Vision, 2(6), 467-479. doi:10.1167/2.6.4

Carter, L. F., \& Schooler, K. (1949). Value, need, and other factors in perception. Psychological Review, 56(4), 200-207. doi:10.1037/ h0060745

Engelmann, J. B., \& Pessoa, L. (2007). Motivation sharpens exogenous spatial attention. Emotion, 7(3), 668-674. doi:10.1037/1528-3542.7.3.668

Firestone, C., \& Scholl, B. J. (2015). Cognition does not affect perception: Evaluating the evidence for "top-down" effects. Behavioral and Brain Sciences, 4629(2016), 1-77. doi:10.1017/ S0140525X15000965

Garner, D. M. (2004). Eating Attitudes Test (EAT-26): Scoring and interpretation. Http://Www.Eat-26.Com/Scoring.Php. http://doi.org/ Retrieved from: http://www.eat-26.com/downloads.php 
Gu, J., Liu, Y., Kyritsis, A. P., \& Bondy, M. L. (2009). Molecular epidemiology of primary brain tumors. Neurotherapeutics, 6(3), 427. doi: 10.1016/j.nurt.2009.05.001

Hollitt, S., Kemps, E., Tiggemann, M., Smeets, E., \& Mills, J. S. (2010). Components of attentional bias for food cues among restrained eaters. Appetite, 54(2), 309-313. doi:10.1016/j.appet.2009.12.005

JASP Team. (2016). JASP (Version 0.8.0.0)[Computer software].

Kral, T., Roe, L., \& Rolls, B. (2004). Combined effects of energy density and portion size on energy intake in women. The American Journal of Clinical Nutrition, 79(6), 962-968. Retrieved from https://ajcn. nutrition.org/content/79/6/962.full

Lazarus, R. S., Yousem, H., \& Arenberg, D. (1953). Hunger and perception. Journal of Personality, 21(3), 312-328. doi:10.1111/j.14676494.1953.tb01774.x

Loewenstein, G. (1996). Out of control : Visceral influences on behavior. Organizational Behavior and Human Decision Processes, 65(3), 272-292. doi:10.1006/obhd.1996.0028

Mogg, K., Bradley, B. P., Hyare, H., \& Lee, S. (1998). Selective attention to food-related stimuli in hunger: Are attentional biases specific to emotional and psychopathological states, or are they also found in normal drive states? Behaviour Research and Therapy, 36(2), 227237. doi:10.1016/S0005-7967(97)00062-4

Montagna, B., Pestilli, F., \& Carrasco, M. (2009). Attention trades off spatial acuity. Vision Research, 49(7), 735-745.

Namdar, G., Avidan, G., \& Ganel, T. (2015). Effects of configural processing on the perceptual spatial resolution for face features. Cortex, 72, 115-123. doi:10.1016/j.cortex.2015.04.007
Neimeijer, R. A. M., de Jong, P. J., \& Roefs, A. (2013). Temporal attention for visual food stimuli in restrained eaters. Appetite, 64, 5-11. doi:10.1016/j.appet.2012.12.013

Piech, R. M., Pastorino, M. T., \& Zald, D. H. (2010). All I saw was the cake. Hunger effects on attentional capture by visual food cues. Appetite, 54(3), 579-582. doi:10.1016/j.appet.2009.11.003

Ptacek, R., Stefano, G. B., Weissenberger, S., Akotia, D., Raboch, J., Papezova, H., ... Goetz, M. (2016). Attention deficit hyperactivity disorder and disordered eating behaviors: Links, risks, and challenges faced. Neuropsychiatric Disease and Treatment, 12, 571.

Radel, R., \& Clement-Guillotin, C. (2012). Evidence of motivational influences in early visual perception: Hunger modulates conscious access. Psychological Science, 23(3), 232-234. doi:10.1177/ 0956797611427920

Tajfel, H. (1957). Value and the perceptual judgment of magnitude. Psychological Review, 64(3), 192-204. doi:10.1037/h0047878

Veltkamp, M., Aarts, H., \& Custers, R. (2008). Perception in the service of goal pursuit: Motivation to attain goals enhances the perceived size of goal-instrumental objects. Social Cognition, 26(6), 720-736. doi:10.1521/soco.2008.26.6.720

Witt, J. K., \& Dorsch, T. E. (2009). Kicking to bigger uprights: Field goal kicking performance influences perceived size. Perception, 38(9), 1328-1340. Retrieved from http://www.ncbi.nlm.nih.gov/pubmed/ 19911630 